\title{
Development of a Critical Care Response - Experiences from Italy During the Coronavirus Disease 2019 Pandemic
}

\author{
Emanuele Rezoagli, MD, PhD ${ }^{a, b}$ * , Aurora Magliocca, MD, PhD ${ }^{a}$, \\ Giacomo Bellani, MD, PhD ${ }^{a, b}$, Antonio Pesenti, $\mathrm{MD}^{\mathrm{c}, \mathrm{d}}$, \\ Giacomo Grasselli, MD ${ }^{c, d}$
}

\section{KEYWORDS}

- Critical care • Pandemic • Coronavirus disease $19 \bullet$ COVID19 Lombardy Network

- Organizational response $\bullet$ Helmet continuous positive airway pressure

- Awake proning

\section{KEY POINTS}

- Italy was the first western country to face a large coronavirus disease 2019 (COVID-19) outbreak.

- COVID19 Lombardy Network responded to the surge of hospital admissions in Northern Italy; it organized a rapid increase in intensive care unit (ICU) beds and implemented measures for containment.

- Scientific evidence was provided by Italian centers to characterize the clinical history of COVID-19 associated respiratory failure.

- Relevant experience was collected in Italy during the pandemic about the use of noninvasive continuous positive airway pressure and awake proning, which were implemented to manage respiratory failure out of the ICU setting.

- Recommendations from national guidelines were structured to guide health care providers on resource allocation; promotion of awareness among Italian citizens within specific humanitarian and educational programs was implemented.

Funded by: CRUI2020.

a Department of Medicine and Surgery, University of Milano-Bicocca, Via Cadore, 48, Monza

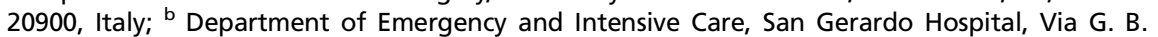
Pergolesi, 33, Monza 20900, Italy; ' Department of Pathophysiology and Transplantation, University of Milan, Via Francesco Sforza 35, Milano 20122, Italy; ${ }^{d}$ Department of Anesthesia, Intensive Care and Emergency, Fondazione IRCCS Ca' Granda Ospedale Maggiore Policlinico, Via della Commenda, 10, Milano 20122, Italy

* Corresponding author. Department of Medicine and Surgery, University of Milano-Bicocca, Via Cadore 48, Monza (MB) 20900, Italy.

E-mail address: emanuele.rezoagli@unimib.it 


\section{INTRODUCTION}

Italy was the first western country facing an outbreak of coronavirus disease 2019 (COVID-19). ${ }^{1}$ The first Italian patient diagnosed with COVID-19 was admitted, on Feb. 20, 2020, to the intensive care unit (ICU) in Codogno Hospital (Lodi, Lombardy, Italy), and the number of reported positive cases increased to 36 in the next 24 hours, and then exponentially for 18 days. This triggered a prompt, coordinated response of the ICUs in the epicenter region of the outbreak that resulted in a massive surge in the ICU bed capacity. ${ }^{2}$

An Italian registry from 3 northern Italian regions (Lombardy, Emilia-Romagna and Veneto) showed that the rate of ICU admission was $12.6 \%$ of COVID-19 hospital admissions. Eight hundred and five patients were admitted and treated in the ICU among 6378 patients hospitalized for COVID-19 in the period between Feb. 24 through March $8,2020 .^{3}$ The coordination of a critical care response in Italy happened in collaboration with out-of-hospital, and out-of-ICU management of patients with respiratory failure.

Furthermore, as part of the implementation of an organizational response to the SARS-CoV2 outbreak, many Italian research groups collected data and provided scientific evidence to understand how to better defeat coronavirus, and make this information quickly publicly available to help other countries that would have to face a similar challenge.

\section{DEVELOPMENT OF A CRITICAL CARE RESPONSE - CORONAVIRUS DISEASE 2019 LOMBARDY INTENSIVE CARE UNIT NETWORK ORGANIZATIONAL PERSPECTIVE}

The critical care response to the COVID-19 pandemic started with the formation of an emergency task force on Feb. 21, created by the Lombardy region authorities and health care representatives: the COVID-19 Lombardy ICU Network (2). The aim of the COVID-19 Lombardy ICU Network was to manage the allocation of resources for all COVID-19 patients requiring ICU treatment in the region. The intensive care team of the Policlinico Maggiore Hospital in Milan led the clinical task force, which was active 24 hours per day, 7 days per week to manage bed request calls.

The 2 primary goals of the network in the initial response phase were to increase surge ICU capacity and to implement measures for containment.

\section{Increase of Surge Intensive Care Unit Capacity}

The precrisis ICU capacity was

- Lombardy: approximately 738 ICU beds (7.4 beds/100,000 people, equal to $2.9 \%$ of the total number of hospital beds)

- Italy: approximately 4682 ICU beds ${ }^{4}$

An exponential model for the prediction of ICU admission rate estimated a need of up to 2500 ICU beds in only 1 week for COVID-19 patients. ${ }^{5}$ Using this model, the whole Italian National Health System would be saturated by mid-April. Drawing from the experience of the Venous-Venous ECMO Respiratory Failure Network, ${ }^{6}$ one of the first initiatives of the network was to create 15 COVID-19 dedicated hub hospitals, with specific expertise in the management of patients with acute respiratory distress syndrome (ARDS) and infectious diseases.

Specific tasks of the hub hospitals were to:

1. Create dedicated ICU cohorts for COVID-19 patients

2. Create triage areas with the possibility to assist critical patients waiting for diagnostic test results for COVID-19 
3. Establish local protocols for triage and rapid allocation of patients with respiratory symptoms

4. Ensure adequate personal protective equipment (PPE) availability and training of health care workers

5 . Immediately notify the regional coordinating center of every confirmed case of critical COVID-19

Through a central coordination of the ICU Network, 130 ICU beds dedicated to COVID-19 patients were created in Lombardy in 48 hours. After the saturation of the designated hub hospitals, almost all hospitals of the region created dedicated ICUs, and on April 2, the ICU capacity reached 1750 beds. In addition, on March 31, 2020, the Milan Fair COVID-19 Intensive Care Hospital was inaugurated. The project, developed by Fondazione Fiera Milano in partnership with Lombardy Region consisted of a temporary hospital with up to 250 ICU beds developed in 20 days, and covering more than 25,000 square meters (Fig. 1). The hospital reorganization process, with the opening of newly dedicated ICUs, has been a multidisciplinary effort, with the involvement of health care providers, hospital managers, and political authorities. ${ }^{7-9}$ The Italian government allocated 845 million euros to the National Health System to ensure a progressive increase of the number of ICU beds for invasive mechanical ventilation, up to $14 \%$ of the total hospital beds. ${ }^{10}$

\section{Implementation of Measures for Containment}

The government instituted extraordinary measures for containment: restrictions within lockdown areas (red zones) were implemented gradually, and then expanded to the entire country on March 9, 2020, until May 18, 2020. A second wave of infections is currently ongoing in several European countries, including Italy. Measures for containment and restrictions within Italian territory were instituted again from Oct. 26, 2020,

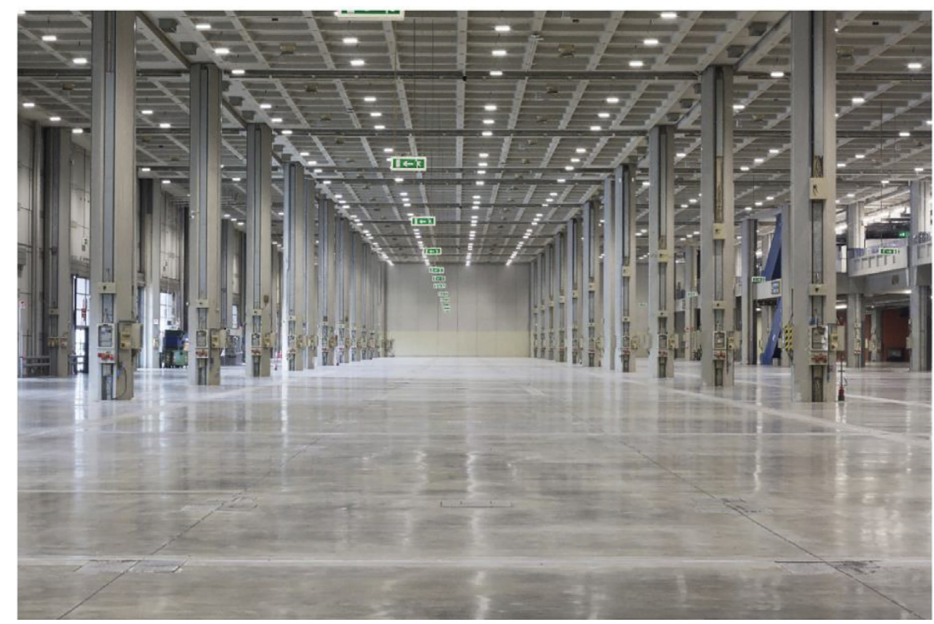

Fig. 1. Representation of the area dedicated to the management of COVID-19 patients at the Fair Milan Covid-19 Intensive Care Hospital covering more than 25,000 square meters of area Portello Pavilions 1 and 2 at Fieramilanocity, Milan, Italy. The image represents the empty space before Fair Milan Covid-19 Intensive Care Hospital was yet staged (permission obtained to reproduce the image by Fondazione Fiera - All Rights reserved - https:// www.ospedalefieramilano.it/it/l-progetto.html). 
based on the estimate of transmissibility within each region. ${ }^{11}$ As of Dec. 13, 2020, the number of hospitalized patients in Lombardy and Italy was 5873 and 30,893, respectively; the number of ICU patients was 714 and 3,158, respectively. Overall, during the last 8 months, totals of 23,810 and 64,520 patients have died of SARS-CoV2 in Lombardy (Fig. 2A,B) and Italy (Fig. 2C,D), respectively.
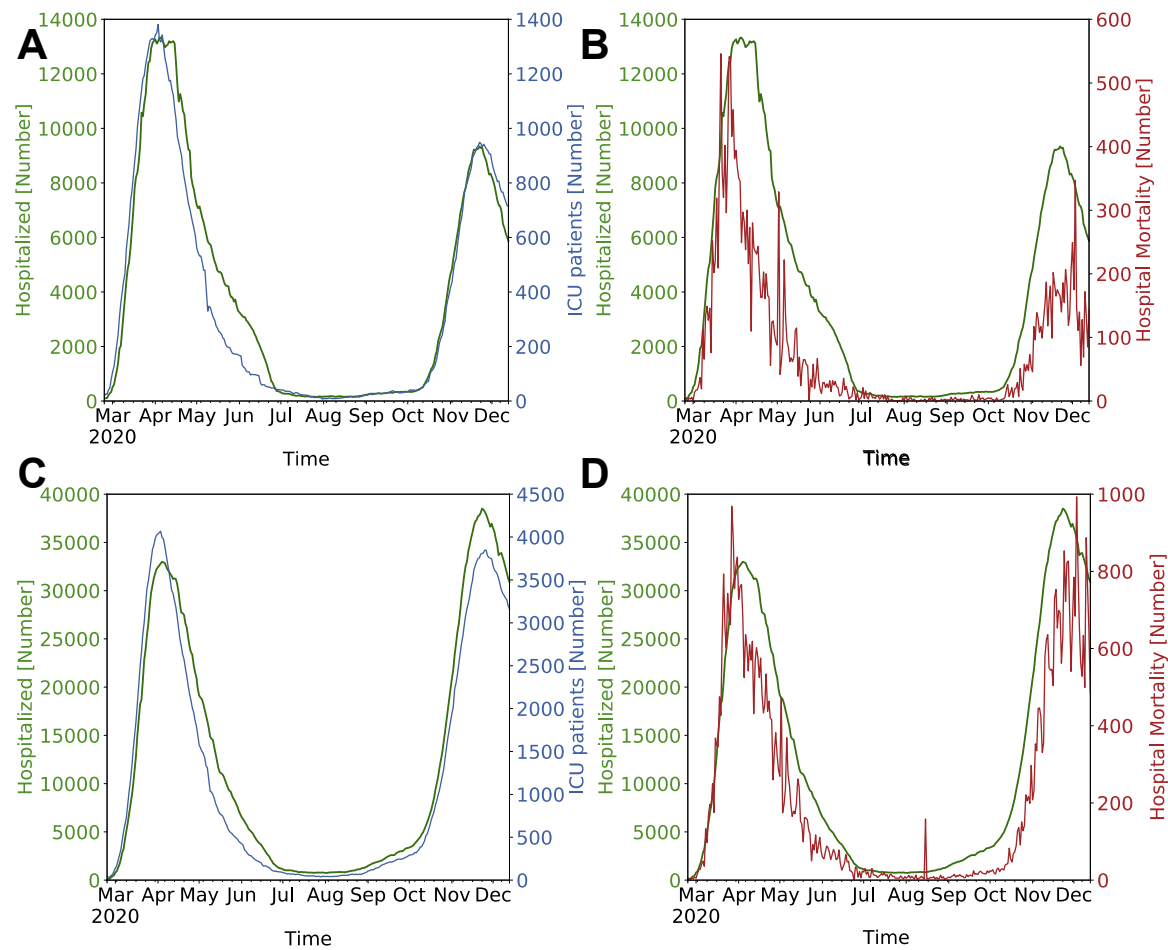

Fig. 2. Number of daily hospital versus ICU admissions $(A, C)$ and hospital admissions versus hospital mortality $(B, D)$ during the Italian first and second wave of SARS-CoV2 outbreak in Lombardy (top panels) and in Italy (bottom panels) from Feb. 24 to Dec. 13, 2020 (original data reports from the public source of "Presidenza del Consiglio dei Ministri - Dipartimento della Protezione Civile" https://github.com/pcm-dpc/COVID-19/blob/master/dati-regioni/dpccovid19-ita-regioni.csv). (D) The peak of mortality reported on Aug. 15 was explained by internal verification of mortality data of Azienda Unità Sanitaria Locale of Parma (Emilia Romagna) that reported 154 deaths over March, April, and May that were not previously included. The distribution of hospitalized patients, ICU admissions, and deaths was different during the 2 peaks of the Italian SARS-CoV2 pandemic. In Lombardy, while the highest number of deaths during the first wave (ie, 546 deaths) was reported approximately 10 days before (ie, on March 20) the highest number of hospitalized patients (ie, 13,328 on April 4) and ICU admissions (ie, 1381 on April 3), during the second coronavirus peak, the highest capacitance in terms of hospital and ICU beds (ie, 9340 and 949, respectively) was reached earlier (ie, on Nov. 22), and contrary to the first wave, 10 days in advance compared with the highest number of deaths (ie, 347 on Dec. 3). Accordingly, in the whole country, a similar date was observed. During the first SARS-CoV2 wave, the highest number of deaths (ie, 969 deaths) was reported about 10 days before (ie, onMarch 26) compared with the highest request of hospital (ie, 33,004 on April 4) and ICU beds (ie, 4068 on April 3). In contrast, during the second peak of the pandemic, the highest numbers of hospital and ICU admissions (ie, 38,507 and 3848, respectively) were recorded on Nov. 23 and 25, respectively, about 10 days before the peak of COVID-19 deaths (ie, 993 on Dec. 3). (Visual courtesy of Francesco Casola.) 


\section{OUT-OF-HOSPITAL CORONAVIRUS DISEASE 2019 RESPONSE - AN ORGANIZATIONAL PERSPECTIVE \\ Organization of the Emergency Medical Service}

The Emergency Medical Services (EMS) of the Lombardy region had to deal with an unprecedented increase in telephone calls to 112 (European emergency number) after the announcement of the first COVID-19 positive patient in Italy on Feb. 20, 2020. Call volumes registered a $264 \%$ increase compared with the 3 previous years on the $23 \mathrm{rd}$ of February in the metropolitan area of Milan (SOREU metropolitan). ${ }^{12}$ Similar reports from other areas showed an increase in calls up to $440 \%$ compared with the preCOVID-19 period. $^{13}$

Several callers were just requesting information and guidance about COVID-19. Many others were suspected symptomatic patients deserving a prompt evaluation of respiratory symptoms, home isolation, and domicile SARS-CoV-2 testing or hospitalization. To cope with the escalation of calls, a COVID-19 response team was instituted by the EMS of the metropolitan area of Milan. ${ }^{14}$ The team, composed of 10 health care professionals and 2 technicians, worked 24 hours per day 7 days per week in assessing the clinical condition of screened individuals to determine the need for hospital admission, or for home testing for SARS-CoV-2 and subsequent isolation. In essence, patients were screened for fever and any respiratory symptoms in order to

\section{Organize ambulance}

dispatch and hospitalization in case of moderate or severe respiratory symptoms

2. Counsel, record, and isolate suspected or confirmed COVID-19 cases with mild symptoms

Despite efforts to maintain ordinary EMS activities through the creation of the COVID-19 response team, the reorganization of the 112 emergency response system, and the implementation of the staff, recent data showed that EMS arrival times were significantly higher compared with the same period in 2019 in Milan, ${ }^{11}$ and in other provinces of Lombardy and Veneto, particularly for time-dependent conditions like out-of-hospital cardiac arrest. ${ }^{15,16}$

The Lombardy EMS coped with a dramatic increase in events caused by the outbreak in the region in an extremely short timeframe, and in a limited area, as occurred in the province of Bergamo. Data about the events managed by the dispatch center for the EMS of Brescia and Bergamo describe a devastating scenario. Fagoni and colleagues reported an increase of $50 \%$ in the number of events managed in March to April 2020, compared with the same period in 2019, with a tenfold increase in the number of the so-called respiratory or infective events. An alarming increase in the number of deaths was reported: $+246 \%$ (odds ratio [OR] 1.7, $P<.0001$ ) in March to April 2020, compared with 2019. ${ }^{17}$ This high mortality was in line with other reports from Italian cities severely affected by the COVID-19 pandemic in northern Italy. ${ }^{18}$

\section{The Challenging Experience of Out-of-Hospital Cardiac Arrest During the Coronavirus Disease 2019 Outbreak}

An almost $60 \%$ increase in out-of-hospital cardiac arrest (OHCA) incidence, coupled with a reduction in the short-term outcomes during the COVID-19 outbreak, was observed in Italy for the first time. ${ }^{15}$ Specifically, during the first 40 days of the COVID-19 pandemic (Feb. 21 through March 31, 2020), the number of OHCAs occurring in the provinces of Lodi, Cremona, Pavia, and Mantua, increased up to $58 \%$ compared with the same period in 2019. An increase in the number of OHCA was 
seen in all 4 provinces, with a worrisome peak in the 2 most afflicted by COVID-19 infection: Lodi (+187\%) and Cremona (+143\%).

Among different etiologies, medical causes were more represented in OHCA during the COVID-19 pandemic. Age and sex of the patients were similar in the 2 study periods, but in 2020 home location and unwitnessed OHCA were more frequent compared with 2019. A decrease in bystander cardiopulmonary resuscitation rate of $15.6 \%$ was observed compared with the 2019 period. The median arrival time of emergency medical service was 3 minutes longer in 2020 than in 2019, and the incidence of out-ofhospital death was almost 15\% higher in 2020 than in 2019. The cumulative incidence of out-of-hospital cardiac arrest in 2020 was strongly associated with the cumulative incidence of COVID-19. The authors then expanded the analysis to the following 60 days after the first COVID-19 patient was isolated, replicating the same results reported for the first 40 days. ${ }^{19}$ On the contrary, a report from Padua in Veneto (northeast of Italy), did not highlight an increase in OHCA incidence and mortality. ${ }^{16}$ However, in line with previous findings, the authors reported an increased EMS arrival time of 1.2 min in 2020 compared with 2019. Interestingly, when they broke the total arrival time into its main components (ie, call to dispatch, dispatch to departure, and departure to arrival), an increase in the time between the call and EMS departure was observed. The authors suggest that the longer call-to-departure time of the EMS could be due to the time spent to investigate COVID-19 status, while the delay in ambulance departure could be explained by PPE procedures and requirements.

\section{IN-HOSPITAL CORONAVIRUS DISEASE 2019 RESPONSE - BUILDING SCIENTIFIC EVIDENCE \\ Intensive Care Unit Management of Coronavirus Disease 2019 Respiratory Failure}

The COVID-19 Lombardy ICU Network was created to promptly respond to the SARSCoV2 outbreak in Italy, and to manage the exponential surge of patients with respiratory failure, needing respiratory support in ICU. Fondazione IRCCS Ca' Granda Ospedale Maggiore Policlinico in Milan was the coordinating center of COVID-19 Lombardy ICU Network, which connected all the ICUs in the Lombardy region. Dedicated staff in the coordinator center of this consortium performed at least 2 telephone calls every day to obtain real-time granular information on most clinical characteristics and outcomes of patients admitted to the ICU. ${ }^{20-22}$

Despite the massive clinical and logistical efforts, COVID-19 Lombardy ICU Network was able to collect and provide scientific evidence about clinical characteristics, risk factors, pathophysiology, and prognosis of patients with SARS-CoV2 induced lung injury. Data collection was not limited to the mentioned phone calls, but also by local granular data collection in a centralized eCRF.

One of the aims of the COVID-19 Lombardy ICU Network was to deliver knowledge as rapidly as possible on a disease still poorly described, ultimately to help other countries facing a similarly dramatic health care experience. ${ }^{23}$ Essentially, the research commitment of Ospedale Maggiore Policlinico was twofold in its objectives:

1. To build a registry that included all epidemiologic, clinical, and prognostic information of adult patients admitted to the hospital from the onset of the pandemic.

2. To create a biobank of samples to perform translational studies.

Data from this registry for national and international researchers will benefit patient care worldwide. ${ }^{24}$ The authors summarized in Table 1 the main scientific evidence reported by the COVID-19 Lombardy ICU Network, together with other Italian investigators, during the pandemic outbreak. 


\section{Table 1}

Scientific evidence provided by the COVID-19 Lombardy ICU Network together with other Italian investigators during the pandemic outbreak to characterize the clinical history of critically ill COVID-19 patients

\section{Areas of}

Research

Clinical

characteristics

of COVID-19

ICU patients
Group of Research

COVID-19 Lombardy

ICU Network ${ }^{20}$
Patient Population

1591 critically ill COVID-19 patients
Time of Inclusion

Feb. 20 to March 8, 2020

\section{Main Findings}

- Median age of 63 (IQR 56-70)

- Male-to-female ratio 4:1

- Hypertension was the most

common comorbidity (49\% of cases)

- Of 1300 patients with ventilator data, $88 \%$ on mechanical ventilation, $11 \%$ on noninvasive ventilation

- Median PEEP $=14 \mathrm{cmH}_{2} \mathrm{O}(\mathrm{IQR}$ 12-16) -median $\mathrm{PaO}_{2} / \mathrm{FiO}_{2}=160$ (114-220)

- Median $\mathrm{Fio}_{2}=70$ (IQR 50-80)

- Prone positioning was used in $27 \%$ of 875 patients

- Patients with hypertension compared to patients without hypertension - were older, with a more severe ARDS, requiring higher levels of PEEP and

showing a higher ICU mortality (38 vs $22 \%$, overall mortality

$26 \%$ )

- Short-term follow-up and half of patients with complete data at follow-up (March 25, 2020) were still in ICU

(continued on next page) 


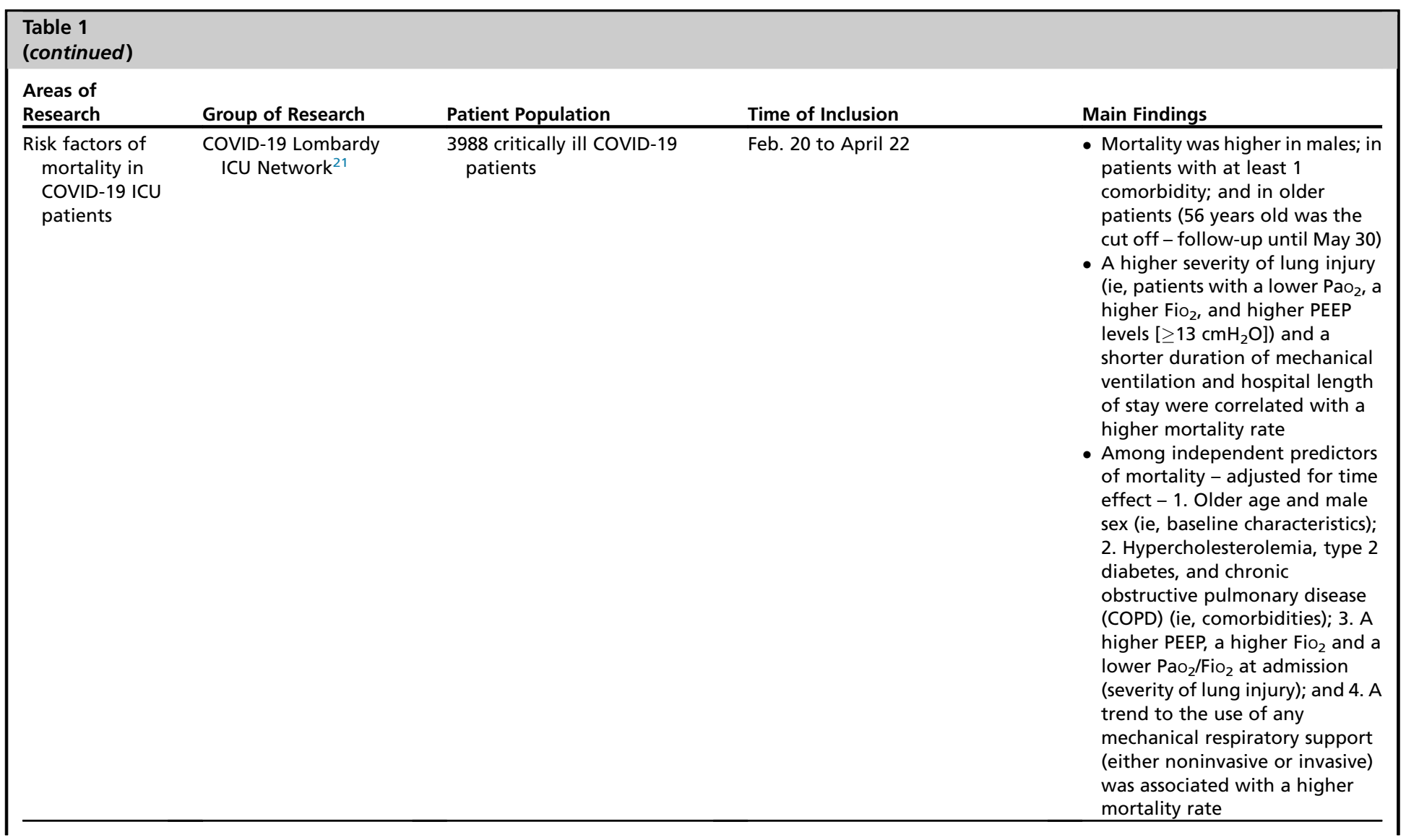


Pathophysiology of COVID-19

ARDS patients
301 critically ill COVID-19 patients
March $9-22$

- Prospective multicente observational study conducted

in different regions from north

to south of Italy

- Median respiratory system compliance was $9 \mathrm{~mL} / \mathrm{cmH}_{2} \mathrm{O}$ higher in COVID-19 associated ARDS compared to patients with ARDS unrelated to COVID-19

- Lung injury associated to COVID-19 appeared not only to be characterized by a parenchymal damage but included also an endothelial injury

- The study reported a strong association between D-dimer concentration and areas of pulmonary hypoperfusion that was assessed by computed tomography (CT)-pulmonary angiography in a subgroup of patients

- The role of different combination of levels of respiratory system compliance and D-dimer on outcome was investigated - in a multivariate model adjusted for sex, age, and severity of ARDS using $\mathrm{PaO}_{2} / \mathrm{FiO}_{2}$ the group of patients at the higher risk of mortality was the one with the worse epithelial and endothelial lung injury, as suggested by the combination of high D-dimer concentration and low compliance of the respiratory system

(continued on next page) 


\begin{tabular}{|c|c|c|c|c|}
\hline \multicolumn{5}{|l|}{$\begin{array}{l}\text { Table } 1 \\
\text { (continued) }\end{array}$} \\
\hline $\begin{array}{l}\text { Areas of } \\
\text { Research }\end{array}$ & Group of Research & Patient Population & Time of Inclusion & Main Findings \\
\hline $\begin{array}{l}\text { Hematological } \\
\text { characteristics } \\
\text { of COVID-19 } \\
\text { patients }\end{array}$ & $\begin{array}{l}\text { - Angelo Bianchi } \\
\text { Bonomi Hemophilia } \\
\text { and Thrombosis } \\
\text { Center in Milan } \\
\text { (COHERENT project) }{ }^{25} \\
\text { - Angelo Bianchi } \\
\text { Bonomi Hemophilia } \\
\text { and Thrombosis } \\
\text { Center in Milan }\end{array}$ & $\begin{array}{l}\text { - } 62 \text { COVID-19 patients - with } \\
\text { low, intermediate or high } \\
\text { intensity of care } \\
\text { - } 24 \text { critically ill COVID-19 } \\
\text { patients }\end{array}$ & $\begin{array}{l}\text { First peak of the Italian COVID-19 } \\
\text { outbreak }\end{array}$ & $\begin{array}{l}\text { Both studies - according to the } \\
\text { analyses of laboratory } \\
\text { biomarkers of pro and } \\
\text { anticoagulation, together with } \\
\text { data regarding the viscoelastic } \\
\text { properties of blood of COVID-19 } \\
\text { patients by the use of } \\
\text { thromboelastography - do not } \\
\text { support hematological } \\
\text { characteristics of disseminated } \\
\text { intravascular coagulation - in } \\
\text { contrast they demonstrated the } \\
\text { presence of a prothrombotic } \\
\text { phenotype that leads to a } \\
\text { procoagulant imbalance that } \\
\text { originates from a complex } \\
\text { interplay between the } \\
\text { inflammatory insult, hemostasis, } \\
\text { and endothelial cells } \\
\text { perturbation }\end{array}$ \\
\hline
\end{tabular}




\section{Double patient ventilation with a single ventilator - feasible and ethical?}

During a pandemic, there may be an imbalance between the numbers of critically ill patients requiring invasive ventilation, and the numbers of mechanical ventilators that are available. An interesting option that was proposed almost 15 years ago by Neyman and Irvin is to connect multiple patients to a single ventilator in order to compensate for the equipment shortage. ${ }^{27}$ Researchers from Milano and Bologna in Italy successfully tested the feasibility of using a single turbine ventilator to provide ventilation in 2 simulated patients with different respiratory mechanic characteristics. ${ }^{28}$ Beitler and colleagues took this experience to the next level and provided evidence of feasibility in COVID-19 patients with ARDS who shared ventilators for at least 2 days, under rigorous protocols, and experienced no adverse events. ${ }^{29}$ This strategy still remains experimental. Critical points still need to be addressed such as the matching of respiratory mechanic characteristics of patients ventilated with a single ventilator - in order to avoid harm in one of them - and the safety of prolonged ventilator sharing.

\section{Out of Intensive Care Unit Management of Coronavirus Disease 2019 Respiratory Failure}

\section{Noninvasive ventilation - state-of-the-art and guidelines}

As stated, the massive burden of SARS-CoV2 on the Italian health care system quickly saturated the availability of ICU beds and mechanical ventilators. Among several, one of the challenges for health care providers was to manage and contain severe intrahospital respiratory failure outside the critical care environment. Noninvasive ventilation allowed physicians to stabilize patients, avoiding the progression to severe hypoxemia and muscle exhaustion that would eventually require invasive mechanical ventilation. Noninvasive positive pressure oxygenation strategies have been recently confirmed to be associated with a lower mortality risk compared to standard oxygen therapy. ${ }^{30}$

Noninvasive positive pressure ventilation (NIPPV) has played a key role in the management of COVID-19 patients out of the ICU during the Italian crisis surge. The rapid guidelines of the European Society of Intensive Care Medicine suggested on 1 side high-flow nasal cannula (HFNC) and NIPPV as strategies to reduce the need for intubation and overcome shortages of mechanical ventilators; on the other side, NIPPV was suggested for invasive ventilation as a last option in a scenario of a shortage of standard full-featured ventilators. ${ }^{31}$ The worldwide guidelines on the management of critically ill COVID-19 patients confirmed the suggestion of the implementation of HFNC and NIPPV in acute hypoxemic respiratory failure (AHRF) and recommended early intubation in a controlled setting if worsening occurred. ${ }^{32}$ The potential increase of virus aerosolization with NIPPV remains a significant concern regarding transmission of infection to health care providers. ${ }^{33}$

\section{The Italian helmet continuous positive airway pressure experience during the coronavirus disease pandemic}

The Italian approach to noninvasive ventilator management of COVID-19 AHRF was characterized in northern Italy by the use of helmet continuous positive airway pressure (c-PAP), because of the large Italian experience in the management of AHRF with this interface. ${ }^{34}$

The helmet is an interface of utmost utility in a pandemic scenario, in order to avoid the risk of aerosolization when helmet NIPPV is delivered through a ventilator, as suggested by Cabrini and colleagues ${ }^{35}$ However, the use of helmet c-PAP has an excellent performance simply with a free-flow generator and a positive end-expiratory pressure (PEEP) valve at the helmet outlet, combined with a high-efficiency particulate 
air (HEPA) filter at the helmet outlet to reduce the risk of environmental contamination. ${ }^{31,36}$ Furthermore, the helmet c-PAP bundle was proposed to optimize patient comfort using

1. A heat and moisture exchanger (HME) filter to decrease incoming noise

2. Counterweight fixing systems to stabilize the helmet position

3. Heated wire tubing with active humidification ${ }^{37}$

Early consensus management of non-ICU patients with SARS-CoV2 in Italy suggested the use of helmet c-PAP without humidification as the first choice. ${ }^{38}$ Three Italian studies have reported data on the use of helmet c-PAP and NIPPV out of a critical care environment in COVID-19 patients. $^{39-41}$

1. In a multicenter observational prospective study, Aliberti and colleagues described the characteristics and the outcome of patients undergoing c-PAP treatment in 3 high-dependency units in 2 Italian hospitals in Milan during the first pandemic wave. Out of 157 patients, helmet c-PAP successfully improved oxygenation from a $\mathrm{PaO}_{2} / \mathrm{FiO}_{2}=143$ to 206 . However, intubation or death was higher compared to non-COVID-19 patients with the same severity of AHRF (45\% vs $23 \%)$. Interestingly, patients with c-PAP failure showed higher inflammation (eg, high interleukin [IL]-6 levels) and activation of the coagulation cascade (eg, high D-Dimer levels) compared with patients who did not fail helmet c-PAP. ${ }^{39}$

2. In the emergency department of Papa Giovanni XXIII hospital (HPG23) from Bergamo (a city overly affected by the surge), Duca and colleagues described patient characteristics and the ventilator management. In a time frame of 10 days, the authors reported that out of 611 patients admitted to the emergency department with suspected COVID-19, 99 received ventilator support (12\% invasive and $88 \%$ noninvasive) in the emergency department, and 85 of them were confirmed positive to SARS-CoV2 (median age 70 years, median $\mathrm{PaO}_{2} / \mathrm{Fio}_{2}$ ratio $=128$ ). Given the resource limitation in the ICU setting at the outbreak onset, the internal hospital protocol in the emergency department of HPG23 adopted the use of helmet C-PAP or NIPPV in the presence of hypoxemia $(\mathrm{SpO} 2<90 \%)$ or RR greater than $30 /$ min during the administration of oxygen therapy by non-rebreather mask with an oxygen flow of $15 \mathrm{~L} / \mathrm{min}$. Patients were admitted to the ward until availability of an ICU bed. The follow-up mortality 2 months later was $77 \%$, which was potentially explained by the severity of hypoxemia of patients admitted to the emergency department with standard oxygen therapy already maximized. ${ }^{40}$

3. The results from the largest data set that described the prevalence and the clinical characteristics of patients with COVID-19 treated with NIV outside the ICU, and that explored the factors associated with NIV failure (defined as need of intubation or death) were reported by Bellani and colleagues in a prospective single-day prevalence study (WARd-COVID). In 31 centers within the COVID-19 Lombardy ICU Network 8753 COVID-19 patients were present, accounting for an average of $62 \%$ of the overall hospital beds. Of these, 909 subjects $(10.4 \%)$ received NIV out of the ICU. The use of the helmet or face-mask was used in a ratio of 3:1. NIV failed in 300 patients (37.6\%). A higher c-reactive protein and lower $\mathrm{PaO}_{2} /$ $\mathrm{FiO}_{2}$ and platelet counts were independent predictors of NIV failure. Mortality rate was $25 \%$ at 60 -day follow-up. Although with a large sample size and the multicentric design of the study, the lower rates of NIV failure and mortality in the WARdCOVID - compared with previous reported studies ${ }^{39,40}$ - may be explained by the different timing of patient enrollment and data collection (ie, 1 month later that the Italian SARS-CoV2 outbreak). At that time, the organizational optimization 
of the ICU resources was already implemented by the COVID-19 Lombardy ICU Network. This consisted of an exponential increase of the number of ICU beds that might have allowed to treat patients with a less severe acute hypercapnic respiratory failure (AHRF) on the ward at the moment of patient enrollment (average $\left.\mathrm{PaO}_{2} / \mathrm{FiO}_{2}=168\right) .{ }^{41}$ An exemplary image representing the use of helmet c-PAP in prone positioning - as performed in the authors' Institutions in Monza and Milano - is provided in Fig. 3.

The Italian experience with prone-positioning in spontaneously breathing patients As with ARDS from other causes, ${ }^{42}$ COVID-19 guidelines propose cycles of 12 to 16 hours of prone positioning in patients with moderate-severe ARDS and undergoing mechanical ventilation, ${ }^{33}$ based on strong physiologic rationale. ${ }^{43,44}$ No information was provided on the use of proning in awake, non-intubated patients in the recent guidelines where the knowledge of the benefits is limited. Nonsystematic differences have been reported in prone positioning compared to supine positioning in healthy volunteers, with the presence of a more homogeneous perfusion in selected subjects that might improve ventilation/perfusion matching. ${ }^{45}$ The use of PEEP has been described to increase the ventilation/perfusion ratio in the dorsal areas of healthy subjects. ${ }^{46}$ However, little is known in terms of the physiologic effects of PEEP in patients undergoing prone positioning with a severe impairment of gas exchange, as in the case with COVID-19 related ARDS.

Italy pioneered the use of prone positioning in awake COVID-19 patients spontaneously breathing and explored the role of noninvasive ventilation during protonation outside the ICU.

1. In a prospective study, Coppo and colleagues explored the feasibility and physiologic effects of prone positioning in 56 patients - on supplemental oxygen therapy only $(21 \%)$ or with helmet c-PAP $(79 \%)$. Prone positioning was feasible in $84 \%$ of patients. Oxygenation was significantly improved in the prone position (average $\mathrm{PaO}_{2} / \mathrm{FiO}_{2}, 286$ vs $\left.181, P<.0001\right)$, and the oxygenation gain was maintained in $50 \%$ of the patient population after resupination. Among other factors, prone positioning seemed more effective if applied early after hospital admission. ${ }^{47}$

2. Data from a retrospective study by Ramirez and colleagues reported that pronation was feasible outside the ICU. Furthermore, patient mobilization, which included prone positioning, was effective in reducing failure rates of C-PAP in COVID-19 patients. ${ }^{48}$

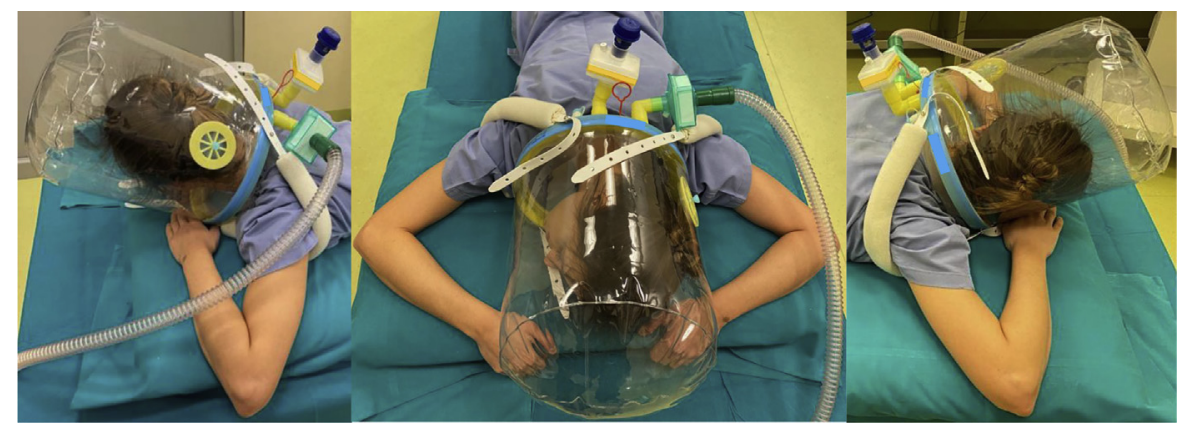

Fig. 3. Exemplary image of continuous positive pressure ventilation delivered by a helmet c-PAP during prone positioning in a healthy volunteer as per the authors' practice at San Gerardo Hospital, Monza and Policlinico Maggiore Hospital, Milano. 
3. Bastoni and colleagues reported a series of 10 patients who met criteria for proning. In 6 patients, with a median $\mathrm{PaO}_{2} / \mathrm{FiO}_{2}$ of $68 \mathrm{~mm} \mathrm{Hg}$, prone positioning was effective at increasing oxygenation to a median $\mathrm{PaO}_{2} / \mathrm{FiO}_{2}$ of $97 \mathrm{~mm} \mathrm{Hg} 1$ hour after. $^{49}$

4. The use of both prone and lateral positioning for 1 hour has been tested in 26 COVID-19 patients during helmet c-PAP admitted to the high-dependency unit of Policlinico Hospital in Milan. Retucci and colleagues observed that the success rate (ie, oxygenation improvement) of proning was higher compared with the use of lateral positioning. However, the short duration of patient positioning may have contributed to the loss of the beneficial improvement of oxygenation when patients returned to the semiseated position. ${ }^{50}$

\section{In-hospital interplay and differences in the use of critical care resources between critical and noncritical care environments}

Three regions in northern Italy joined together in a common effort to build a large network that included different experiences from the part of Italy that was severely hit by the outbreak. This led to the development of the COVID-19 Northern Italian ICU Network, which strived to report scientific evidence on the management and patient characteristics from different Italian areas.

In an interesting analysis from the COVID-19 Northern Italian ICU Network, the investigators reported differences among patients managed in and out of the ICU during the first 14 days of the pandemic outbreak (Feb. 24 to March 8, 2020). In the ICU, bed capacity rapidly increased from 1545 to 1989 beds (28.7\%). In data obtained in 802 patients within 14 days, the percentage of patients who received respiratory support increased from $0.6 \%$ to $37 \%$ out of the ICU. Patients were located in the infectious disease ward, pneumology ward, emergency medicine, and intermediate care unit, with a proportion of $47 \%, 31 \%, 15 \%$, and $7 \%$, respectively. The proportion of patients admitted to ICU decreased from $20.3 \%$ to $15.2 \%$. Patients located out of the ICU, compared to within the ICU, had more comorbidities, received more oxygen therapy and NIV, (with the exception of c-PAP that did not differ between the 2 groups), had higher $\mathrm{PaO}_{2} / \mathrm{FiO}_{2}$ and $\mathrm{pH}$, and lower respiratory rate, $\mathrm{PacO}_{2}$, and base excess. ${ }^{3}$

A useful score to predict clinical deterioration (defined as escalation of care to the ICU or death) in COVID-19 patients was proposed by Cecconi and colleagues. Higher levels of C-reactive protein (CRP) and creatinine, together with the presence of coronary artery disease, higher degree of hypoxemia, and a respiratory rate above or equal to 20 breaths per minute were used to build a prognostic index with a high predictive accuracy (85\%) and easy implementation at bedside. ${ }^{51}$ The findings obtained by Cecconi and coworkers were confirmed by the CORIST study - including almost 4000 patients from 30 clinical centers from northern, central and southern Italy - in which elevated CRP, impaired renal function, and advanced age predicted in-hospital mortality. ${ }^{52}$

\section{SURGICAL PROCEDURES - CORONAVIRUS DISEASE 2019 POSITIVE AND NEGATIVE PATIENTS}

The hospital overload of COVID-19 patients led to a sudden and unplanned interruption of elective surgical activities that led to the difficult process of balancing between the risk of delaying a cancer diagnosis and treatment, versus suffering a potential COVID-19 exposure, An individualized approach, based on a case-by case evaluation, is suggested. ${ }^{53}$ Furthermore, in COVID-19-positive patients, precise, well-established plans and protocols must be implemented to perform emergent and nondeferrable surgical procedures. ${ }^{54}$ 


\section{NATIONAL GUIDELINES ON RESOURCE ALLOCATION DURING CORONAVIRUS DISEASE 2019 - THE RESPONSE OF THE ITALIAN SOCIETY OF ANESTHESIA, ANALGESIA, RESUSCITATION, AND INTENSIVE CARE}

The Italian Society of Anesthesia, Analgesia, Resuscitation, and Intensive Care (SIAARTI) provided documents and recommendations to manage the SARS-CoV2 outbreak at different levels, including both clinical practice ${ }^{55}$ and ethical considerations. ${ }^{56,57}$

From an ethical perspective, SIAARTI elaborated recommendations in a scenario in which the surge of critical patients admitted to the hospital created an "imbalance between the real clinical needs of the population and the effective availability of intensive resources." The society highlighted 3 principles that should guide the decision-making process for appropriate allocation of limited health care resources:

1. Clinical appropriateness

2. Proportionality of care

3. Distributive justice

The SIAARTI guidelines aimed to help the clinicians in managing the potential emotional burden associated with resource allocation and make explicit the criteria for resource allocation. Although individual judgment must be considered part of the clinical decision, the presence of national recommendations served as a guide for clinicians to avoid frank disparities in the judgment and an arbitrary perspective in the presence of dramatic choices.

Scarce resources should be evaluated and considered in the presence of a higher probability of survival and of saved years of life - evaluating patient age, comorbidities, and the functional status before the event - and aiming to achieve a better outcome for the highest number of people. ${ }^{56,57}$ Ethical and legal nuances of the national recommendations have been provided by the SIAARTI. ${ }^{58,59}$

SIAARTI, with the collaboration of Società Italiana di Infermieri di Terapia Intensiva (ANIARTI), Società Italiana di Medicina di Emergenza e Urgenza (SIMEU), Società Italiana di Cure Palliative (SICP), also provided guidance to health care workers for the management of communication on patient clinical conditions to the families that were completely isolated during the lockdown, and could not have any visual or physical interaction with their relative admitted to the hospital. The document, shared by these 4 societies, had 3 components, including a statement on communication with families, the key points used to develop the statements, and a checklist with instructions for how to make appropriate phone calls. ${ }^{60}$

Communication of clinical information to patient families was made difficult not only by the severity and acuity of such a novel disease, but also by the fear of the disease in non-health care workers, which created situations where health care professionals were praised while they were at work, but experienced discrimination when outside of the hospital setting. ${ }^{61}$ This may have contributed to a psychological effect in the frontline personnel of the SARS-CoV2 pandemic. ${ }^{62}$

To promote awareness among people about the clinical condition of patients, and the daily working conditions of the health care workers during the COVID outbreak in Italy, Hope Onlus was created in collaboration with the Ospedale Maggiore Policlinico in Milan, and promoted the project "\#Covid-19 con Hope" \#Covid-19@storiedisperanza (www.hopeonlus.org). This important project has an educational and cultural mandate to explain the impact of COVID-19 on the society to Italian citizens within a humanitarian program of Hope Onlus, at both national and international levels. This project is composed of photo exhibitions, including images of the real-life conditions in the hospitals, together with stories of the health care workers in action (Fig. 4). The 


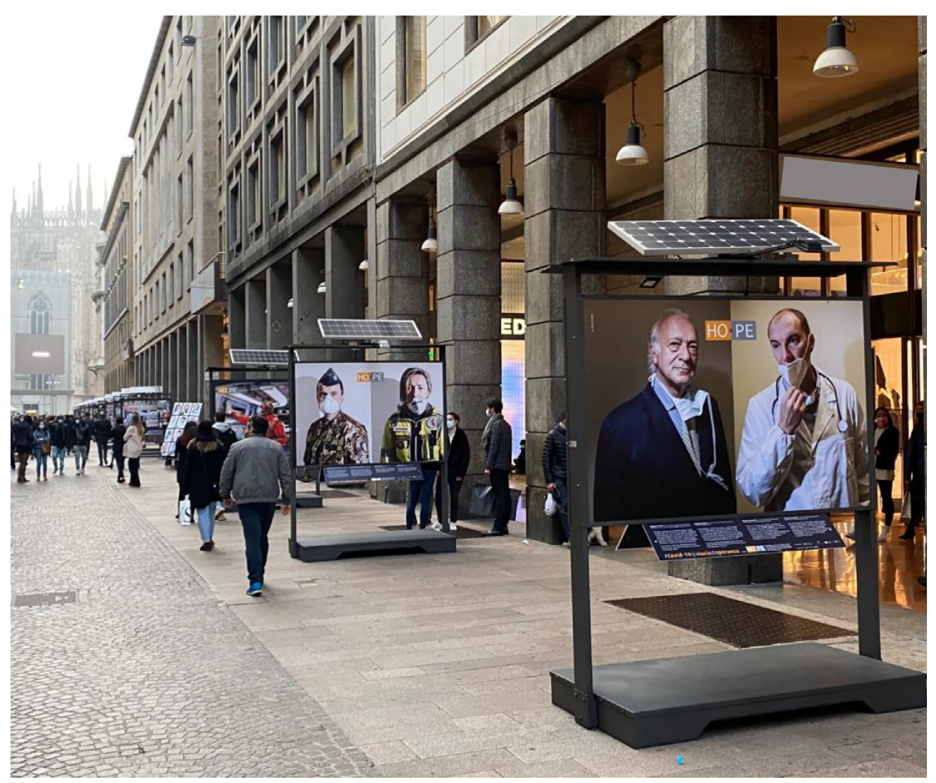

Fig. 4. Humanitarian Program Hope Onlus "\#Covid-19 con Hope" \#Covid-19@storiedisperanza. On the first stand, Prof. Antonio Pesenti, on the left, and Prof. Giacomo Grasselli, on the right - Clinical Director and Clinical Lead of the Intensive Care Unit of Policlinico Maggiore Hospital, Milano - the 2 main actors who led the Lombardy Crisis Unit and coordinated the COVID-19 Lombardy ICU network.

authors' university is also participating in the FLOWS project, led by the National University of Ireland in Galway, which aims to identify the needs and development of best practice guidance for the psychological support of frontline health care workers during and after COVID-19. ${ }^{63}$

In conclusion, the Italian critical care experience during the first wave of the COVID19 pandemic was a pioneer example of an organizational and clinical response to the outbreak. At the same time, a continuous effort was made to provide scientific evidence to understand how to better defeat coronavirus, and make this information available to help other countries worldwide.

\section{CLINICS CARE POINTS}

- Italy was the first western country to face a large COVID-19 outbreak.

- COVID19 Lombardy Network responded to the surge of hospital admissions in the Northern Italy; it organized a rapid increase in ICU beds and implemented measures for containment.

- Scientific evidence was provided by Italian centers to characterize the clinical history of COVID-19 associated respiratory failure

- Relevant experience was collected in Italy during the pandemic about the use of noninvasive continuous positive airway pressure and awake proning, which were remarkably implemented to manage respiratory failure out of the ICU setting.

- Recommendations from national guidelines were structured to guide health care providers on resource allocation; promotion of awareness among Italian citizens within specific humanitarian and educational programs was implemented 


\section{ACKNOWLEDGMENTS}

The authors thank Francesco Casola, PhD, for his help with the visual of Fig. 2.

\section{DISCLOSURE}

The authors have nothing to disclose.

\section{REFERENCES}

1. WHO Director-General's opening remarks at the media briefing on COVID-19: 11 March 2020. 2020. Available at: https://covid19. who.int/?gclid=CjOKCQiAOeBBhDiARIsADyBcE4_O7cTc98_eNV7hXefnM_DNAfVfCmwKaXImvzHipvRpqTF mrlaGBsaAqOVEALw_wcB.

2. Grasselli G, Pesenti A, Cecconi M. Critical care utilization for the COVID-19 outbreak in Lombardy, Italy: early experience and forecast during an emergency response. JAMA 2020;323(16):1545-6.

3. Tonetti T, Grasselli G, Zanella A, et al. Use of critical care resources during the first 2 weeks (February 24-March 8, 2020) of the Covid-19 outbreak in Italy. Ann Intensive Care 2020;10(1):133.

4. Pecoraro F, Clemente F, Luzi D. The efficiency in the ordinary hospital bed management in Italy: An in-depth analysis of intensive care unit in the areas affected by COVID-19 before the outbreak. PLoS One 2020;15(9):e0239249.

5. Remuzzi A, Remuzzi G. COVID-19 and Italy: what next? Lancet 2020;395(10231): 1225-8.

6. Patroniti N, Zangrillo A, Pappalardo F, et al. The Italian ECMO network experience during the 2009 influenza $A(H 1 N 1)$ pandemic: preparation for severe respiratory emergency outbreaks. Intensive Care Med 2011;37(9):1447-57.

7. Fagiuoli S, Lorini FL, Remuzzi G, et al. Adaptations and Lessons in the Province of Bergamo. N Engl J Med 2020;382(21):e71.

8. Carenzo L, Costantini E, Greco M, et al. Hospital surge capacity in a tertiary emergency referral centre during the COVID-19 outbreak in Italy. Anaesthesia 2020;75(7):928-34.

9. Buoro S, Di Marco F, Rizzi M, et al. Papa Giovanni XXIII Bergamo Hospital at the time of the COVID-19 outbreak: Letter from the warfront. Int J Lab Hematol 2020; 42(Suppl 1):8-10.

10. Available at: https://www.gazzettaufficiale.it/eli/id/2020/03/09/20G00030/sg. Accessed December 13, 2020.

11. Cori A, Ferguson NM, Fraser C, et al. A new framework and software to estimate time-varying reproduction numbers during epidemics. Am J Epidemiol 2013; 178(9):1505-12.

12. Marrazzo F, Spina S, Pepe PE, et al. Rapid reorganization of the Milan metropolitan public safety answering point operations during the initial phase of the COVID-19 outbreak in Italy. J Am Coll Emerg Physicians Open 2020;1(6):1240-9.

13. Perlini S, Canevari F, Cortesi S, et al. Emergency department and out-of-hospital emergency system (112-AREU 118) integrated response to coronavirus disease 2019 in a northern Italy centre. Intern Emerg Med 2020;15(5):825-33.

14. Spina S, Marrazzo F, Migliari M, et al. The response of Milan's emergency medical system to the COVID-19 outbreak in Italy. Lancet 2020;395(10227):e49-50.

15. Baldi E, Sechi GM, Mare C, et al. Out-of-hospital cardiac arrest during the covid19 outbreak in Italy. N Engl J Med 2020;383(5):496-8. 
16. Paoli A, Brischigliaro L, Squizzato T, et al. Out-of-hospital cardiac arrest during the COVID-19 pandemic in the Province of Padua, Northeast Italy. Resuscitation 2020;154:47-9.

17. Fagoni N, Perone G, Villa GF, et al. The Lombardy emergency medical system faced with COVID-19: the impact of out-of-hospital outbreak. Prehosp Emerg Care 2020;25:1-7.

18. Piccininni M, Rohmann JL, Foresti L, et al. Use of all cause mortality to quantify the consequences of covid-19 in Nembro, Lombardy: descriptive study. BMJ 2020;369:m1835.

19. Baldi E, Sechi GM, Mare C, et al. COVID-19 kills at home: the close relationship between the epidemic and the increase of out-of-hospital cardiac arrests. Eur Heart J 2020;41(32):3045-54.

20. Grasselli G, Zangrillo A, Zanella A, et al. Baseline characteristics and outcomes of 1591 patients infected with SARS-CoV-2 Admitted to ICUs of the lombardy region, Italy. JAMA 2020;323(16):1574-81.

21. Grasselli G, Greco M, Zanella A, et al. Risk factors associated with mortality among patients with COVID-19 in intensive care units in Lombardy, Italy. JAMA Intern Med 2020;180(10):1-11.

22. Grasselli G, Tonetti T, Protti A, et al. Pathophysiology of COVID-19-associated acute respiratory distress syndrome: a multicentre prospective observational study. Lancet Respir Med 2020;8(12):1201-8.

23. Cook DJ, Marshall JC, Fowler RA. Critical illness in patients with COVID-19: mounting an effective clinical and research response. JAMA 2020;323(16): 1559-60.

24. Bandera A, Aliberti S, Gualtierotti R, et al. Response of an Italian reference institute to research challenges regarding a new pandemic: COVID-19 network. Clin Microbiol Infect 2020;S1198-743X(20):30374-8.

25. Peyvandi F, Artoni A, Novembrino C, et al. Hemostatic alterations in COVID-19. Haematologica 2020. https://doi.org/10.3324/haematol.2020.262634.

26. Panigada M, Bottino N, Tagliabue P, et al. Hypercoagulability of COVID-19 patients in intensive care unit: a report of thromboelastography findings and other parameters of hemostasis. J Thromb Haemost 2020;18(7):1738-42.

27. Neyman G, Irvin CB. A single ventilator for multiple simulated patients to meet disaster surge. Acad Emerg Med 2006;13:1246-9.

28. Tonetti T, Zanella A, Pizzilli G, et al. One ventilator for two patients: feasibility and considerations of a last resort solution in case of equipment shortage. Thorax 2020;75(6):517-9.

29. Beitler JR, Mittel AM, Kallet R, et al. Ventilator sharing during an acute shortage caused by the COVID-19 pandemic. Am J Respir Crit Care Med 2020;202(4): $600-4$.

30. Ferreyro BL, Angriman F, Munshi L, et al. Association of noninvasive oxygenation strategies with all-cause mortality in adults with acute hypoxemic respiratory failure: a systematic review and meta-analysis. JAMA 2020;324(1):57-67.

31. Aziz S, Arabi YM, Alhazzani W, et al. Managing ICU surge during the COVID-19 crisis: rapid guidelines. Intensive Care Med 2020;46(7):1303-25.

32. Alhazzani W, Møller MH, Arabi YM, et al. Surviving sepsis campaign: guidelines on the management of critically ill adults with coronavirus disease 2019 (COVID19). Intensive Care Med 2020;46(5):854-87.

33. Hui DS, Chow BK, Ng SS, et al. Exhaled air dispersion distances during noninvasive ventilation via different Respironics face masks. Chest 2009;136:998-1005. 
34. Bellani G, Patroniti N, Greco M, et al. The use of helmets to deliver non-invasive continuous positive airway pressure in hypoxemic acute respiratory failure. Minerva Anestesiol 2008;74(11):651-6.

35. Cabrini L, Landoni G, Zangrillo A. Minimise nosocomial spread of 2019-nCoV when treating acute respiratory failure. Lancet 2020;395(10225):685.

36. Lucchini A, Giani M, Winterton D, et al. Procedures to minimize viral diffusion in the intensive care unit during the COVID-19 pandemic. Intensive Crit Care Nurs 2020;60:102894.

37. Lucchini A, Giani M, Isgrò S, et al. The "helmet bundle" in COVID-19 patients undergoing non invasive ventilation. Intensive Crit Care Nurs 2020;58:102859.

38. Vitacca M, Nava S, Santus P, et al. Early consensus management for non-ICU ARF SARS-CoV-2 emergency in Italy: from ward to trenches. Eur Respir J 2020;55(5):2000632.

39. Aliberti S, Radovanovic D, Billi F, et al. Helmet CPAP treatment in patients with COVID-19 pneumonia: a multicenter, cohort study. Eur Respir J 2020;56(4): 2001935.

40. Duca A, Memaj I, Zanardi F, et al. Severity of respiratory failure and outcome of patients needing a ventilatory support in the Emergency Department during Italian novel coronavirus SARS-CoV2 outbreak: preliminary data on the role of Helmet CPAP and non-invasive positive pressure ventilation. EClinicalMedicine 2020;24:100419.

41. Bellani G, Grasselli G, Cecconi M, et al. Noninvasive ventilatory support of COVID-19 patients outside the Intensive Care Units (WARd-COVID). Ann Am Thorac Soc 2021. https://doi.org/10.1513/AnnalsATS.202008-10800C.

42. Fan E, Del Sorbo L, Goligher EC, et al. An Official American Thoracic Society/European Society of Intensive Care Medicine/Society of Critical Care Medicine clinical practice guideline: mechanical ventilation in adult patients with acute respiratory distress syndrome. Am J Respir Crit Care Med 2017;195(9):1253-63.

43. Gattinoni L, Taccone P, Carlesso E, et al. Prone position in acute respiratory distress syndrome. Rationale, indications, and limits. Am J Respir Crit Care Med 2013;188(11):1286-93.

44. Guerin C, Baboi L, Richard JC. Mechanisms of the effects of prone positioning in acute respiratory distress syndrome. Intensive Care Med 2014;40(11):1634-42.

45. Musch G, Layfield JDH, Harris RS, et al. Topographical distribution of pulmonary perfusion and ventilation, assessed by PET in supine and prone humans. J Appl Physiol (1985) 2002;93(5):1841-51.

46. Mure M, Nyrén S, Jacobsson $\mathrm{H}$, et al. High continuous positive airway pressure level induces ventilation/perfusion mismatch in the prone position. Crit Care Med 2001;29(5):959-64.

47. Coppo A, Bellani G, Winterton D, et al. Feasibility and physiological effects of prone positioning in non-intubated patients with acute respiratory failure due to COVID-19 (PRON-COVID): a prospective cohort study. Lancet Respir Med 2020;8(8):765-74.

48. Ramirez GA, Bozzolo EP, Castelli E, et al. Continuous positive airway pressure and pronation outside the intensive care unit in COVID 19 ARDS. Minerva Med 2020. https://doi.org/10.23736/S0026-4806.20.06952-9.

49. Bastoni D, Poggiali E, Vercelli A, et al. Prone positioning in patients treated with non-invasive ventilation for COVID-19 pneumonia in an Italian emergency department. Emerg Med J 2020;37(9):565-6. 
50. Retucci M, Aliberti S, Ceruti C, et al. Prone and lateral positioning in spontaneously breathing patients with COVID-19 pneumonia undergoing noninvasive helmet CPAP Treatment. Chest 2020;158(6):2431-5.

51. Cecconi M, Piovani D, Brunetta E, et al. Early predictors of clinical deterioration in a cohort of 239 patients hospitalized for COVID-19 infection in Lombardy, Italy. J Clin Med 2020;9(5):1548.

52. Di Castelnuovo A, Bonaccio M, Costanzo S, et al. Common cardiovascular risk factors and in-hospital mortality in 3,894 patients with COVID-19: survival analysis and machine learning-based findings from the multicentre Italian CORIST Study. Nutr Metab Cardiovasc Dis 2020;30(11):1899-913.

53. Moletta L, Sefora Pierobon E, Capovilla G, et al. International guidelines and recommendations for surgery during COVID-19 pandemic: a systematic review. Int J Surg 2020;79:180-8.

54. Coccolini F, Perrone G, Chiarugi M, et al. Surgery in COVID-19 patients: operational directives. World J Emerg Surg 2020;15(1):25.

55. Sorbello M, El-Boghdadly K, Di Giacinto I, et al. The Italian coronavirus disease 2019 outbreak: recommendations from clinical practice. Anaesthesia 2020; 75(6):724-32.

56. Vergano M, Bertolini G, Giannini A, et al. SIAARTI recommendations for the allocation of intensive care treatments in exceptional, resource-limited circumstances. Minerva Anestesiol 2020;86(5):469-72.

57. Vergano M, Bertolini G, Giannini A, et al. Clinical ethics recommendations for the allocation of intensive care treatments in exceptional, resource-limited circumstances: The Italian perspective during the COVID-19 epidemic. Crit Care 2020;24:165.

58. Piccinni M, Aprile A, Benciolini P, et al. [Ethical, deontologic and legal considerations about SIAARTI document "clinical ethics recommendations for the allocation of intensive care treatments, in exceptional, resource-limited circumstances." Recenti Prog Med 2020;111(4):212-22.

59. Sulmasy DP. Principled decisions and virtuous care: an ethical assessment of the SIAARTI Guidelines for allocating intensive care resources. Minerva Anestesiol 2020;86(8):872-6.

60. Multidisciplinary Working Group. ComuniCovid." Italian Society of Anesthesia and Intensive Care (SIAARTI), Italian Association of Critical Care Nurses (Aniarti), ItalianSociety of Emergency Medicine (SIMEU), and Italian SocietyPalliative Care (SICP). How to communicate with families of patients in complete isolation during SARS-CoV-2 pandemic multidisciplinary working group "ComuniCoViD. Recenti Prog Med 2020;111(6):357-67.

61. Cabrini L, Grasselli G, Cecconi M, et al. Yesterday heroes, today plague doctors: the dark side of celebration. Intensive Care Med 2020;46(9):1790-1.

62. Azoulay E, De Waele J, Ferrer R, et al. Symptoms of burnout in intensive care unit specialists facing the COVID-19 outbreak. Ann Intensive Care 2020;10(1):110.

63. Available at: https://hrbopenresearch.org/articles/3-54. Accessed December 13, 2020. 